\title{
CCD PHOTOMETRY OF GALACTIC GLOBULAR CLUSTERS V. NGC 2808
}

\author{
Alistair R. Walker \\ Cerro Tololo Inter-American Observatory, National Optical Astronomy Observatories ${ }^{1}$
}

\begin{abstract}
We present a deep color magnitude diagram for the galactic globular cluster NGC 2808 (C0911-646), reaching 3 magnitudes below the main sequence turnoff. A set of local photometric standards are provided, and the mean cluster reddening and metallicity are constrained using several photometric techniques. The unusual horizontal branch morphology discovered by Sosin et al. (1997, ApJ, 480, L35) is confirmed. No significant radial population gradients are identified to within the limits of the data. We investigate the redwards color spread on the main sequence found by Ferraro et al. (1997, A\&A, 327, 598) in a field centered 6.3 arcmin from the cluster center, attributed to binaries. We conclude that the spread is in the most part due to a non-Gaussian distribution of photometric errors about the main sequence ridgeline.
\end{abstract}

\section{INTRODUCTION}

NGC 2808 (C0911-646) is a prominent southern galactic globular cluster situated at low galactic latitude $\left(l=282 .{ }^{\circ} 19, b=-11 .{ }^{\circ} 25\right)$. It was first noticed by Harris $(1974,1975)$ that the horizontal branch (HB) morphology is very unusual, with the red HB (RHB) stars clearly separate from a group of very blue HB (BHB) stars. In a follow-up study (Harris 1978) the photometry was placed on a more secure basis and he suggested that the most plausible explanation for the two groups of stars was one whereby mass segregation of some $0.05-0.1 M_{\odot}$ had occurred. His observations were confirmed in subsequent color magnitude diagram (CMD) studies by Ferraro et al. (1990) and by Byun \& Lee (1993). Gratton \& Ortolani (1986) and Buonanno, Corsi, \& Fusi Pecci (1989) extended the CMD to below the main sequence (MS) turn-off. A multi-color study by Alcaino et al. (1990) (hereafter A90) also reached below the MS but did not include HB stars; all these deep studies used

\footnotetext{
1 Operated by the Association of Universities for Research in Astronomy, Inc., under cooperative agreement with the National Science Foundation.
} 
small-format CCDs and single fields thus few evolved stars were included in their CMDs. Clement \& Hazen (1989) found only two RR Lyrae variables despite the evident richness of the total HB population. Sosin et al. (1997) (hereafter S97) used the WFPC2 on the Hubble Space Telescope to prepare a deep CMD for the center of NGC 2808 in the $B$ and $V$ bands. They also observed with an ultraviolet (F218W) filter, providing for the first time good color and hence temperature resolution for the BHB stars. The BHB was found to extend to far fainter magnitudes in the $V, B-V$ CMD than was previously known, well below the level of the MS turn-off. This long blue tail has two extra gaps, which are narrow and well-defined, their width corresponding to $\sim 0.01 M_{\odot}$ from comparison with $\mathrm{HB}$ evolutionary models (Dorman, Rood \& O'Connell 1993). They discuss possible explanations for the HB multi-modality, which include mass loss, composition variations, and dynamical effects, but conclude that none of these explanations are completely satisfactory. This discussion has been extended by Ferraro et al. (1998) to other clusters that also have extended blue HB . Finally, Ferraro et al. (1997) (hereafter F97) present a $V, V-I$ CMD of a $4 \times 4$ arcmin region near NGC 2808, which reaches to $V \sim 23.5$ and demonstrate that the MS has dispersion greater on the redward side of the ridge-line than on the blue side. This is interpreted as being due to a large fraction $(\sim 24 \%)$ of binaries.

The CMD morphology exhibited by clusters such as NGC 2808 challenges our understanding of the advanced stages of the evolution of low-mass stars, and how much

the dense stellar environment of globular clusters affects these processes. As the extreme example, NGC 2808 is especially important. The present observations complement those made with HST WFPC2, by covering the whole cluster field at a higher photometric accuracy than that achieved by earlier ground-based studies. The faintest magnitude in the less crowded regions of the cluster is deeper than the HST photometry, however interpretation of the CMD here is complicated by the crowding, by the significant contamination due to field stars, and by variable extinction.

\section{OBSERVATIONS}

A series of CCD images centered on NGC 2808 was taken on the night of 1996 December 12, using the 4-m Blanco telescope at prime-focus. They consisted of $V$ band (6 x $5 \mathrm{~s}, 5 \times 50 \mathrm{~s}, 5 \times 300 \mathrm{~s})$ and $B$ band $(4 \times 10 \mathrm{~s}, 4 \times 60 \mathrm{~s}, 4 \times 400 \mathrm{~s})$ integrations using SITe 2048 CCD \#4, and filters from set \#1. The CCD was read out through all four amplifiers simultaneously with an Arcon controller which delivered a read time of 35s, thus observing efficiency was over $75 \%$ despite the many short exposures. The 6-element prime focus corrector, which includes prisms providing correction for atmospheric dispersion, produces 
excellent quality images over an area several times greater than the 15 arcmin square field

provided by the CCD. The pixel size of 24 microns corresponds to 0.432 arcsec and produces rather poorly sampled images even in the worse than median seeing of this night (image fwhm 1.1 arcsec). Additionally, the CCD is not flat, however to first order the curvature was compensated by use of a weak negative lens as dewar window. The particular lens installed slightly under-corrected the curvature, causing a slow degradation in focus as a function of field angle. The night was photometric.

NGC 2808 was also observed on 1996 December 17 with the CTIO 0.9-m telescope and Tektronix 2048 \#3 CCD, through 3 x 3 inch $B$ and $V$ filters (set Tek 2). This CCD was also read out through all four amplifiers with an Arcon controller. Three sets of exposures were taken with differing integration times (30s, 100s and 300s in $V$; 50s, 150s and 450s in $B)$, these frames were used for checking the photometric zeropoints, as described in detail below. Seeing was 1.0-1.2 arcsec and the night was photometric. Pixel size at the 0.9-m telescope is 0.40 arcsec.

The frames were processed in the usual way, with overscan subtraction and trimming, followed by subtraction of a zero level exposure and removal of the instrument signature by division using twilight sky flat-field exposures. The zero and flat calibration frames were each made up from five individual exposures, combined so as to remove stars from the flats and cosmic rays from both flats and zeroes. Tests at the telescope using the illuminated dome "white-spot" were used to measure the CCD linearity and determine the shutter characteristics. The CCDs were measured to be linear to better than $0.5 \%$ (laboratory tests demonstrate linearity at the $0.1 \%$ level) over their operating range. Differential non-linearity between different CCD amplifiers is much easier to measure, and is at the $0.1 \%$ level or better for both the CCDs used here. The shutters produce offsets of $+65 \mathrm{~ms}$ (0.9-m) and $+50 \mathrm{~ms}(4-\mathrm{m})$ at their center which must be added to the nominal exposure time, and a gradient such that by the corners of the CCDs the offset is near zero. A mean offset was added to all exposure times, but since all exposures were longer than 5 seconds (for which the error induced amounts to $\pm 0.5 \%$ ) the gradient was disregarded.

\section{DATA REDUCTIONS}

The individual exposures from the 4-m data-set were moved onto common centers and combined to produce short, medium and long exposures in each color. Integration times for the short exposures did not saturate stars at the tip of the cluster red giant branch (RGB) and the resolution is sufficient that bright RGB stars can be measured to the cluster center. However this is a dense cluster, and on the longer exposures the cluster 
center is hopelessly saturated, for these the central regions were blobbed out with all pixels within a radius of 150 arcsec of the cluster center being set to invalid. Reductions then proceeded using DAOPHOT II and ALLSTAR (Stetson 1987, 1995), with the exception that a stand-alone program was written to more critically identify PSF stars than the PICK routine in DAOPHOT. Due to the slight variation in image quality as a function of radius, the PSF used was a linearly varying Moffat function. Between 25000 and 40000 stars were measured on each frame, following two passes through the FIND algorithm. Subtracted frames were clean, in particular the bright star residuals did not differ appreciably with position, demonstrating that the linearly varying PSF was correctly accounting for the spatial variations in image shape. Stars were carefully matched between $V$ and $B$ frames, which included accounting for a slight (approximately $0.05 \%$ ) difference in scale.

The $0.9-\mathrm{m}$ reductions were carried out in almost identical manner to those for the 4-m. The Moffat-function PSF chosen was allowed to vary quadratically as a function of radius, since this telescope is a classical Cassegrain and coma is obtrusive by the corners of the CCD. Stars further than 1000 pixels from the CCD center were not measured because of this degradation in image quality.

\section{CALIBRATIONS}

The 4-m observations used the standard 4-inch square PFCCD $B$ and $V$ filters, for which color terms are accurately known. These were able to be checked, and extinction coefficients measured, by observation of the Landolt (1992) fields SA 98 and PG 1047 prior to the NGC 2808 observations, and the PG 0918 field subsequently. The PG 1047 field was at airmass two, the others at airmass similar to the cluster. Each field contains several stars with very accurate photometry, and a total of 30 stars were measured here, using star aperture and sky annuli radii of 14, 18 and 28 pixels respectively. The fitted color equations are

$V=v+0.019(b-v)$

$B-V=0.901(b-v)$

with extinction coefficients $K_{V}=0.13$ and $K_{B}=0.26$. Standard errors of the $V$ and $B-V$ zeropoints are 0.002 and 0.003 mag. respectively.

Considering now the 0.9-m data-set, a total of 75 standards from Landolt (1992) were measured in these two filters, and also with I filter number 31. The standards were measured using digital aperture photometry, with star aperture and sky annuli of 14, 18 and 28 pixels respectively. The fitted color equations are $V=v+0.014(b-v)$ 
$B-V=0.904(b-v)$

$V-I=1.008(v-i)$

with extinction coefficients $K_{V}=0.111, K_{B}=0.215$, and $K_{I}=0.044$. Standard errors of the zeropoints are 0.002 mag. in each of $V, B-V$ and $V-I$. A discussion of the same telescope-filter-CCD combination is given by Walker (1995), from more extensive standard star observations than here. A conclusion from that study is that errors arising from ignoring small non-linearities in these color equations are negligible for $V$ and $V-I$, while for $B-V$ the non-linearities amount to \pm 0.01 mag or less for $-0.3<B-V<1.8$. It was further supposed from the similarity of the 4-m and 0.9-m CCDs and filter sets that a similar conclusion could be made for the 4-m data. There is no evidence from the present data sets that color equation non-linearities exceed these very small levels, and as such they will be ignored. A caveat is that there are no Landolt standards observed here with colors in the range $-0.2<B-V<0.15$ and we have to assume that the color equation is well-behaved in this region. This is unfortunately precisely the color range of the BHB stars. With this assumption, we conclude that the zeropoints and color equations determined for observations on these two nights should allow transformation to the standard system to an accuracy of $\sim 0.01 \mathrm{mag}$ in both $V$ and $B-V$.

\subsection{NGC 2808 Local Standards}

Local standards were chosen on the $0.9 \mathrm{~m}$ frames, and on the short-exposure $4-\mathrm{m}$ frames, using the criteria of having no significant contaminating stars within 8 arcsec radius, and be bright but not saturated. Most of these stars are of necessity several arcmin distant from the cluster center. However we also included stars that had been measured by A90 which are rather closer to the cluster center. This included six stars with photoelectric photometry from Alcaino \& Liller (1986). On the short 4-m frames two of these stars are saturated, for the remaining four we find that in the mean, in the sense Walker-Alcaino $\Delta V=0.01 \pm 0.02$ $\Delta(B-V)=0.01 \pm 0.01$ Similar results are found from the $0.9-\mathrm{m}$ data, $\Delta V=-0.01 \pm 0.02$ ( $\mathrm{n}=6$ short $),-0.01 \pm 0.03$ ( $\mathrm{n}=4 \mathrm{med}),-0.01 \pm 0.04$ ( $\mathrm{n}=3$ long) $\Delta(B-V)=-0.01 \pm 0.01(\mathrm{n}=6),-0.01 \pm 0.01(\mathrm{n}=4), 0.00 \pm 0.02(\mathrm{n}=3)$.

These results are very satisfactory, however there is a strong caveat. High contrast displays of the NGC 2808 frames clearly show that there are many faint stars within the $\mathrm{r}=14$ pixel star aperture for the stars as close to the cluster center as the A90 standards. 
Thus despite the good agreement for these stars, aperture photometry through relatively large apertures may well be systematically incorrect. The new local standards set up here (Table 1) are all more distant from the cluster and are consequently much less crowded than the A90 stars. Tests were made by subtracting the psf-fitted faint stars adjacent to the local standards, then comparing photometry through a range of apertures for these frames and the originals. This showed that the new local standards have no discernible mean zero-point offset $(\ll 0.01 \mathrm{mag})$ in $V$ and $B-V$ when photometry with and without faint companions are compared. However offsets for the A90 stars are typically a few 0.01 mag when this test is performed.

An extensive set of photoelectric standards were provided by Harris (1978) in his major study of NGC 2808. Most of these stars are too distant to be on the CCD frames here. Nine stars, with $V$ magnitudes between 13.5 and 17.5 do overlap. Excluding one very discrepant star, the mean zeropoint difference between the psf photometry here and Harris' photoelectric photometry is, in the sense Walker-Harris,

$\Delta V=-0.01 \pm 0.02(\mathrm{se}),(\mathrm{n}=8)$

$\Delta(B-V)=0.01 \pm 0.02(\mathrm{se})$.

We made two further aperture photometry comparisons. Firstly, we compared photometry for approximately 20 stars in the magnitude range $V=15-18$ mag that were measured with good signal to noise on all the 0.9-m frames. No systematic differences are found between the results from the short, medium and long exposures, and indeed most individual comparisons agree to within 0.01-0.02 mag per star. Secondly, we also compared aperture photometry for the same 20 stars between the 4-m short exposures and the 0.9-m long exposures. The mean differences are $0.00 \pm 0.01$ in both $V$ and $B-V$. We calculate the formal error by adding in quadrature the mean errors for the calibrations (linearity, flat-fielding, shutter), the errors in color equations, zeropoints and extinction, and finally the errors in the system of local standards. The latter group of errors are always difficult to estimate and are usually dominated by systematic effects. Formally the errors in $V$ and $B-V$ are little more than $0.01 \mathrm{mag}$, but we will double these and assume that the NGC 2808 photometric system is accurate to $\pm 0.02 \mathrm{mag}$ in each of $V$ and $B-V$ for the purposes of the following discussion. The short, medium and long exposure frames were tied together via photometry of many (typically several hundred) overlap stars.

\subsection{Comparison of HB Photometry, and Variable Stars}


At this stage we checked all available blue HB photometry of NGC 2808, since (see below) accurate photometry of the $\mathrm{HB}$ components is critical when photometrically determining the cluster reddening. In particular, we looked at the position of the RHB clump, and the color difference between the RHB clump and the BHB at $V=V(H B)+1.0$ to test whether the color differences between the various studies considered of a zeropoint shift only, or whether they also differ in scale. This comparison is listed in Table 2. Apparently only the Byun \& Lee (1993) photometry, which has never been published in detail, shows both scale and zeropoint differences when compared to the present results. The other studies show only zeropoint shifts to within the errors. Unfortunately these zeropoint shifts are rather large. Applying shifts to the Ferraro et al. (1990) photometry of $\Delta V=+0.11, \Delta(B-V)=-0.05$ mags produces an excellent match for the whole CMD, including the MS, with the exception that the brightest RGB stars are 0.02-0.03 mag redder here. For the S97 photometry, shifts of $\Delta V=0, \Delta(B-V)=+0.05$ mag are required. In this case, after these shifts are applied agreement between the two CMD's is excellent everywhere, including the brightest RGB stars.

The only search for variable stars in NGC 2808 is that by Clement \& Hazen (1989), who from B-band photographic photometry identified two RR Lyrae variables (V12 with period 0.30577705 days and V6 with period 0.5389687 days), a Cepheid (V10 with period 1.76528 days) and two longer period variables (V1, V11). The latter two stars are confirmed to be very red, with photometry here of $V=13.10, B-V=1.85$ and $V=15.11, B-V=1.27$ respectively. $\quad \mathrm{V} 1$ is at the tip of the giant branch, whereas V11 is well down the RGB. For V11, identification is not secure from the small-scale Clement \& Hazen (1989) chart, and there is a very red star with $V=14.64, B-V=1.74$ only 3 arcsec $\mathrm{N}$ which may be the true variable. This latter star lies well to the red of the RGB and is probably a field star. There are a few stars which by virtue of their position in the CMD are RR Lyrae candidates, but they are not numerous by comparison to the total HB population.

\section{METALLICITY AND REDDENING}

Ferraro et al. (1990) provide a list of metallicity and reddening estimates for NGC 2808. The range of $[\mathrm{Fe} / \mathrm{H}]$ is from -0.96 to -1.48 , and of $E(B-V)$ from 0.21 to 0.34 . These are not all strictly comparable, and the widely used compilation by Zinn (1985) lists $[F e / H]=-1.37 \pm 0.09$ and $E(B-V)=0.22$. Rutledge et al. (1997a,b) measured the Calcium triplet feature for many galactic globular clusters; for NGC 2808 their mean value is based on 20 stars and on the Zinn scale is $[\mathrm{Fe} / \mathrm{H}]=-1.36 \pm 0.05$, in excellent agreement with Zinn (1985). 
The situation for the NGC 2808 reddening is not very satisfactory, with a wide range of estimates in the recent literature. A90, via Vandenberg and Bell (1985) isochrones, found $E(B-V)=0.28$ at an adopted $[\mathrm{Fe} / \mathrm{H}]=-1.27$, while S97, using the Holtzman et al. (1995) filter zeropoints and color equations, find that they can only simultaneous fit their UV and visual CMD's with relatively small values for the reddening, between $E(B-V)=0.09$ and 0.16. Schlegel, Finkbeiner \& Davis (1998) find $E(B-V)=0.23$, while Burstein \& Heiles (1984) give $E(B-V)=0.17$. The Schlegel et al. (1998) values, plotted for a radius of $1 \mathrm{deg}$. around NGC 2208 show rather variable reddening, ranging from $E(B-V)=0.17$ to 0.29 , also note that their zeropoint calibration produces reddenings systematically 0.02 mag greater than those of Burstein \& Heiles (1984).

We will proceed by comparing the colors of the HB features and the RGB with those for other clusters. The position of the latter is determined both by the reddening and by the overall metallicity $[M / H]$. There are no high-dispersion spectroscopic analyses available for NGC 2808, or indeed for the comparison clusters we choose below, which would provide $[M / H]$. We make do with measurements based on the infrared Calcium triplet (Rutledge et al. 1997a,b), strictly [Ca/H], and earlier measurements (see Zinn \& West 1984, Zinn 1985) denoted as $[\mathrm{Fe} / \mathrm{H}]$ but based on a number of indicators. This is an unsatisfactory situation, as discussed for example by Rutledge et al. (1997b), unlikely to be improved without more high dispersion spectroscopic analyses for stars in many globular clusters. If at all possible, reddening should be determined by methods that have little sensitivity to the metal abundance.

Unfortunately, the NGC 2808 instability strip boundaries are not well defined thus precluding the use of the color of the blue edge which Walker (1998) confirms to have constant $B-V$ over a wide range of metallicity, nor are there accurate light curves and colors for the RR Lyraes which would allow the use of Sturch's method (Sturch 1966, Walker 1990). Instead we are forced mostly to metallicity-sensitive methods. Sarajedini \& Layden (1997) extend their method for simultaneously determining metallicity and reddening from a $(V, V-I)$ CMD to the $(V, B-V)$ case. Using several galactic globular clusters with well-determined values for these two quantities as standards they provide a calibration based on the color of the RGB at the level of the HB?, and the magnitude difference between the RGB and the $\mathrm{HB}$ at $(B-V)_{0}=1.1$ and 1.2. The fiducial cluster NGC 1851 with HB level measured at the RR Lyrae position, and 47 Tucanae with HB measured from the red HB clump are the two calibrating clusters expected to bracket NGC

\footnotetext{
${ }^{2}$ The level of the HB used is the mean magnitudes of the RR Lyraes if that is known, else the estimated mean magnitude of the HB at the color of the instability strip. For clusters with red HB morphology, the mean magnitude of the RHB clump is utilised (A. Sarajedini, private communication).
} 
2808 in metallicity, so we will for comparison purposes measure the HB level both from the RHB stars and at the position of the RR Lyraes. Since NGC 2808 has very few stars in the vicinity of the instability strip, there is difficulty in estimating $V_{H B}$. We will use our observations of NGC 1851 (Walker 1992, 1998) and NGC 6362 (Brocato et al. 1999, Walker 1999), both of which have $\left\langle V_{R R}>-V_{R H B}=-0.08 \pm 0.01\right.$, where for NGC 6362 we use the bluer RHB clump stars only, due to the increase in $V$ magnitude range for the redder RHB clump stars. Thus for NGC $\left.2808<V_{R R}\right\rangle=16.22$ and $\left\langle V_{R H B}\right\rangle=16.30$. The vertical extent of the RHB clump is $0.2 \mathrm{mag}$, so the ZAHB level for this feature is $V=16.40 \pm 0.02$. Although these various HB levels are all very similar, the slope of the NGC 2808 RGB at the HB level is approximately $\Delta B-V) / \Delta V=-0.15$ and 2.5 mags further up the RGB $\Delta(B-V) / \Delta V=-0.45$. Consequently, small errors in the placement of the HB have little influence on $(B-V)_{0, g}$ but will strongly affect any index involving the upper RGB. An example is the RGB shape calibration provided by Mighell, Sarajedini \& French (1998), who derive expressions for the color difference between the HB and positions 2 mags $\left(S_{-2.0}\right)$ and 2.5 mags $\left(S_{-2.5}\right)$ brighter, as a function of $[\mathrm{Fe} / \mathrm{H}]$.

After iterating to find consistent values from the Sarajedini \& Layden (1997) fitting equations, we find $E(B-V)=0.16 \pm 0.02$ and $[F e / H]=-1.05 \pm 0.1$ (internal errors), using $<V_{R R}>$ to represent the HB level, and $E(B-V)=0.18 \pm 0.02$ and $[\mathrm{Fe} / \mathrm{H}]=-1.15 \pm 0.1$ (internal errors), using $V_{R H B}$ to represent $V_{H B}$. Forcing $[\mathrm{Fe} / \mathrm{H}]$ to a more metal poor value consistent with the spectroscopy would increase the reddening by $\sim 0.03 \mathrm{mag}$, very close to the $E(B-V)=0.20 \pm 0.02$ obtained from the Zinn \& West $(1984)(B-V)_{0, g}$ calibration. Since the method uses a very similar $(B-V)_{0, g}$ relation to that of Zinn \& West (1984) this result is likely telling us that the the determination of the shape of the RGB is responsible for forcing this more metal-rich result. A more metal-rich value is also found from $S_{-2.0}$, $[F e / H]=-1.10$ and -1.17 using $V_{H B}=16.22$ and 16.30 respectively. This may be suggesting that the overall metal abundance $[M / H]$ is higher than indicated by $[\mathrm{Fe} / \mathrm{H}]$, assuming $[\mathrm{Ca} / \mathrm{Fe}]=0$, since it is $[\mathrm{M} / \mathrm{H}]$ rather than $[\mathrm{Fe} / \mathrm{H}]$ that determines the location and shape of the RGB.

We can also compare the CMD to that of NGC 1851 (Walker 1992, 1998), which has $E(B-V)=0.02 \pm 0.02$, and $[F e / H]=-1.29 \pm 0.07$, the latter very similar to that for NGC 2808. The single Ca triplet measurement from an integrated spectrum of the cluster (Armandroff \& Zinn 1988) implies $[\mathrm{Fe} / \mathrm{H}]=-1.16 \pm 0.07$ (Da Costa \& Armandroff 1995) which is more consistent with comparisons between positions of RGB's for various clusters. Despite this uncertainty we will continue with the comparison, since NGC 1851, with bimodal HB, shares the characteristic of unusual HB structure with NGC 2808. NGC 1851 has a very tight clump of RHB stars centered at $V=16.15, B-V=0.66$, whereas the NGC 2808 RHB clump is more diffuse, and is centered near $V=16.30, B-V=0.83$. 
Forcing these to match shows that the NGC 2808 stars are $0.17 \pm 0.03$ redder than the NGC 1851 stars, therefore $E(B-V)=0.19 \pm 0.04$. Comparing the two RGB's gives $E(B-V)=0.19 \pm 0.03$ with no correction applied for the differential difference.

In principle the BHB stars can also be overlaid, which has the advantage that intrinsic color differences due to possibly different metallicity will be minimized. However there are some cautions. For these very blue stars the photometric color calibration may not be as secure as for cooler stars, as discussed section 4. Additionally, any cluster-cluster variation in the luminosity- temperature relationship for these stars means that they will follow different loci in the CMD. Borissova et al. (1997) show that the NGC 6229 and M3 sequences do not agree well, despite near-identical reddening and metallicity, and the same is found here when comparing the NGC 1851 and NGC 2808 BHB stars. Brocato et al. (1998) give a detailed discussion of this problem. More consistent results appear to be obtained if the comparison is restricted to the color of the BHB where the sequence begins its rapid decrease in $(V)$ brightness, which occurs at $(B-V)=0.10 \pm 0.02$ for NGC 1851 and at $B-V=0.30 \pm 0.05$ for NGC 2808, therefore $E(B-V)=0.22 \pm 0.05$ for NGC 2808 . As a consistency check we generated a fiducial from the Borissova et al. (1997) CMD of NGC 6229. This cluster has HB morphology similar to NGC 2808, with well determined and small reddening of $E(B-V)=0.01$, and $[F e / H]=-1.44$. Their CMD covers the $\mathrm{RGB}$ and the HB only, so no comparison is possible for the turn-off region. We normalized the $V$ magnitudes at the RGB, and shifted the NGC 6229 color zeropoint by 0.19 mag to the red, i.e. we have assumed $E(B-V)=0.20$. We find that the RGB of NGC 6229 is approximately 0.02 mag bluer than that of NGC 2808 at the level of the HB, consistent with the nominal difference of approximately $0.1 \mathrm{dex}$ in the metallicities. The slope of the NGC 6229 RGB is steeper, also consistent this cluster being somewhat more metal poor than NGC 2808.

We can also compare with the CMD for NGC 6362 (Brocato et al. 1999), which has $[\mathrm{Fe} / \mathrm{H}]=-1.18 \pm 0.06$ (Rutledge et al. 1997b) and with slightly more metal rich (0.1 dex) earlier estimates, all on the Zinn scale. Reddening, by a variety of methods, is $E(B-V)=0.05 \pm 0.02$. NGC 6362 is one of the most metal-rich clusters with halo kinematics, and has a well-populated HB with many BHB stars, but without the extensive tail of hot BHB stars displayed by NGC 2808. With offsets applied to NGC 6362 fiducials of $\Delta V=0.92$ and $\Delta(B-V)=0.15 \mathrm{mag}$ the fit to the HB is excellent, however the NGC 6362 RGB lies 0.04 mag redder than the NGC 2808 RGB. This is consistent with NGC 2808 being 0.2 dex more metal poor than NGC 6362 .

In summary, there seems little doubt that the NGC 2808 reddening is near $E(B-V)=0.20 \pm 0.02$. The metal abundance, based on Calcium triplet measurements 
(Rutledge at al. 1997b) is $[\mathrm{Fe} / \mathrm{H}]=-1.36 \pm 0.05$, on the Zinn scale. The shape of the RGB is only marginally consistent with these values, suggesting an overall more metal-rich $[M / H]$ and a slightly smaller value for the reddening. There is difficulty matching both the HB and the RGB-MS in a comparison with NGC 1851, but the inconsistency is greatly reduced if the calcium triplet-based metallicity $[\mathrm{Fe} / \mathrm{H}]=-1.16$ is adopted for NGC 1851 .

\section{COLOR MAGNITUDE DIAGRAM MORPHOLOGY}

The CMD morphology has been well-illustrated in the several previous studies of this cluster, as discussed in the introduction, and we have made comparisons with other clusters above. Of most interest is the HST study by S97 which for the first time clearly shows the presence of multiple groups of stars on the $\mathrm{HB}$, and a prominent blue straggler sequence. However even with HST the crowding in the small field near the cluster center is sufficiently great that this CMD only reaches to $V=21$. We therefore begin by preparing a composite CMD from the three sets of exposures. It should be stressed that this diagram (Figure 2), containing 33464 stars, is primarily meant to illustrate the morphology of the various sequences and does not represent in any way the relative numbers of stars in each, since the short exposures reached to the cluster center for the brightest stars whereas for the longer exposures the central regions of the cluster were not measured. It also became apparent that there were significant reddening variations across the field, as the position of the color of the MS turn-off varied when the data were plotted in spatial (eg 512x512 pixels) sections. As an aside, in principle it would have been preferable to use the centroid of the clump of RHB stars to ascertain reddening, but there were insufficient such stars in the outer parts of the frame to provide an accurate centroid. Photometry in the outer parts of the frame were adjusted to match the mean of the center 1024x1024 pixels, the absolute values of the adjustments averaged $0.028 \mathrm{mag}$ for $B-V$ and were all less than 0.055 mag. Variations in reddening across the field are not unexpected given the low galactic latitude and the evidence for clumpy reddening in the region from the Schlegel et al. (1998) values. The latter are consistent with the present results, showing slightly higher reddening to the NE of NGC 2808 and slightly lower reddening in the other three orthogonal directions.

The resultant CMD, which has been corrected for differential reddening, clearly shows the principal sequences, contaminated by a very significant number of field stars. When preparing this diagram it became clear that crowding of this highly condensed cluster is the major factor in preventing accurate ground- based photometry. Even so, the main sequence is well defined and here extends to 4 magnitudes fainter than the turn-off. The HB morphology is, of course, highly unusual, and we confirm the gaps discovered by S97. 
Since their HB terminated near $V=21 B-V=-0.1$ which was their magnitude limit, it is important to see whether or not this really is the end of the HB. The magnitude limit of the present data is $B=25$ and $V=24$ and the $\mathrm{HB}$ end is seen near $V=21.2$, thus this $i s$ indeed the end. There is a range in color for the BHB stars between magnitudes 16-18 that is likely due to star-star differential reddening, also responsible for broadening other vertical sequences with small photometric errors, such as the RGB. The most prominent gap in the $\mathrm{HB}$ is the region of the RR Lyraes. The RHB clump is well defined and is separated by a luminosity gap from stars at the base of the AGB. The subgiant branch and RGB appear normal, with the RGB terminating near $B-V=1.9$.

The RGB bump, arising from a hiatus in the rate of stellar evolution up the RGB as a consquence of the hydrogen burning shell passing through a discontinuity left by the maximum penetration of the convective envelope (Iben 1968), is clearly visible in the CMD. Cassisi \& Salaris (1997) present a critical theoretical discussion of the positions of the ZAHB and the RGB bump, deriving $\Delta V_{H B}^{\text {bump }}=1.083+1.380[M / H]+0.231[M / H]^{2}$, where $[M / H]$ is the global heavy metal abundance, which can be approximated as (Salaris, Chieffi \& Straniero 1993) as $[M / H]=[F e / H]+\log (0.638 \cdot f+0.362)$, where $\log f=[\alpha / F e]$, the enhancement factor for the $\alpha$ elements. They also find that the position of the bump depends on the mass of the model, and derive an increase of $\Delta V_{H B}^{\text {bump }}$ of almost $0.024 \mathrm{mag}$ for an increase of $1 \mathrm{Gyr}$ in age of the cluster. In an observational evaluation, Saviane et al. (1998) approximate the relation as $\Delta V_{H B}^{\text {bump }}=0.66[\mathrm{Fe} / \mathrm{H}]+0.87$. The $\mathrm{HB}$ reference magnitude in both cases is that for the ZAHB in the vicinity of the RR Lyraes. We use the comparison with NGC 1851 and 6362 (see above) to estimate $V_{Z A H B}$ for NGC 2808, thus obtaining $\Delta V_{H B}^{\text {bump }}=0.00 \pm 0.07$, and with the S98 calibration $[\mathrm{Fe} / \mathrm{H}]=-1.32 \pm 0.11$. With the Cassisi \& Salaris (1997) formulation, we find $[M / H]=-0.93 \pm 0.07$. Since there is no measured value of $[\alpha / F e]$ for NGC 2808, we will adopt a value of $0.30 \pm 0.05$, whereupon $[\mathrm{Fe} / \mathrm{H}]=-1.14 \pm 0.08$. As pointed out by Cassisi \& Salaris (1997), their theoretical scale agrees well with high-dispersion spectroscopic measurements of cluster metallicities, which tend to give values somewhat more metal-rich than the Zinn scale, the Zinn-scale estimate of $[\mathrm{Fe} / \mathrm{H}]=-1.36 \pm 0.05$ transforms to $[\mathrm{Fe} / \mathrm{H}]=-1.11 \pm 0.03$ on the Carretta \& Gratton (1997) high dispersion spectroscopic scale (Rutledge et al. 1997b). So in this admittably round-about fashion, the position of the NGC 2808 RGB bump is found to be in excellent agreement with the Cassisi \& Salaris (1997) models, assuming the $[\alpha / F e]$ ratio is normal. This would also argue against enhanced $[\alpha / F e]$ being responsible for the shallower than expected RGB, as discussed above. 


\section{RADIAL POPULATION GRADIENTS}

The measurement of the relative numbers of stars as a function of radius in a cluster as rich as NGC 2808 is a difficult task, and one best suited to HST. Here we have made a few comparisons between groups of stars of roughly equivalent brightness in order to minimize magnitude-dependent completeness corrections. The extent of the difficulty with the present data set is evident by comparing the ratio of $A G B+R G B$ stars brighter than $V=15$ with the RGB stars with $V=15$ to 17.5 , using only the short exposure frames. Outside $r=150$ pixels (one pixel $=0.432$ arcsec) the ratio faint:bright is constant at 8.8, whereas from 50:150 pixels it is 5.4, and inside 50 pixels it is 1.5 . We find no significant differences between the distribution of RHB and BHB stars within the errors, comparing only the brightest clump $(V<18.5)$ of BHB stars; there are $2.1 \pm 0.5$ times as many RHB stars as these BHB stars in the $150-300$ pixel radii annulus and this ratio is preserved,

within a progressively larger error, at greater distances. For the two fainter clumps of BHB stars the number counts would appear to indicate a deficiency of such stars at small radii, compared to the RHB or brighter BHB stars, but a comparison between ratios of MS stars with the same $V$ magnitude shows that the number counts of stars as faint as this are seriously incomplete in the inner annuli. We do find that the HB star distribution in the outer annuli (ie at distances greater than 300 pixels from the cluster center, is similar to that seen at the cluster center by S97. This would argue for there being no gross mass differences between the sub-groups on the HB, or else the cluster is dynamically mixed. The latter could be tested by comparing the radial distribution of the BS stars to that for other cluster stars.

\section{BINARIES}

The F97 field is about 6 arcmin south and 2 arcmin east of the cluster center, and their CMD shows a redwards broadening of the MS that can be interpreted as being due to binaries, with some $24 \pm 4 \%$ of apparently single MS stars being in such a category. As cautioned by F97 these may be unresolved optical binaries, some of which must be expected due to the high star density, rather than true physical binaries. Unfortunately it is difficult to directly test between the two options, as both mass segregation of the binary systems (e.g. Rubenstein \& Bailyn 1999) and the effects of image blending will act to concentrate such stars towards the center of the cluster. F97 consider, from artificial star tests, that no more than $10 \%$ of the candidate binaries are optical in character.

We first compare the two extremes. Stars nearer than 350 pixels (2.52 arcmin) from the cluster center (note that no stars from the long exposures were measured this close) 
are plotted in Figure 3. Of the 3566 stars in this CMD, only 300 are on the MS $(V>20)$ and these clearly suffer much photometric scatter. However there is very little field star contamination and the evolved star sequences, with the exception of the fainter BHB stars, are well populated. There are at least $25 \mathrm{BS}$ stars. But from this CMD nothing can be said about the spread of MS stars. We can contrast this (Figure 4) with a CMD made up by selecting stars more than 1000 pixels from the cluster center. This shows a well defined cluster MS, few evolved stars, and the expected large number of field stars. A careful examination of the distribution of stars about a MS fiducial shows no evidence for any significant binary population. Turning now to fields at intermediate radii, we plot the CMD between radii of $600-800$ pixels (Figure 5) and $800-1000$ pixels (Figure 6). It is visually obvious from the CMDs, especially Figure 5, that there is a redwards spread of the MS. We therefore need to carefully test the stellar image crowding in these annuli in order to differentiate between the optical and physical binary hypotheses. The number of measured stars in each CMD is 9119 and 6753; thus there are 96 and 167 pixels per star respectively. With image fwhm of under 2.5 pixels, this degree of crowding does not seem excessive, and indeed with 1 arcsec seeing the image crowding for these data becomes severe only well inside the $r=600$ pixels radius. To put this initial impression on a more quantitative basis, artificial stars were added to the long-exposure frames using the ADDSTAR routine of DAOPHOT (Stetson 1987). As long as the brightness of the added stars exceeded the MS magnitude limit of $V \sim 23.5$ by more than one magnitude, the percentage of stars recovered in both annuli exceeded 95\%. It is therefore clear, as was concluded by F97, that optical binaries make only a minor contribution to the observed distribution. Our next test was to restrict the analysis to only the stars in the $600-800$ pixel annulus. Note that this annulus is slightly closer in the mean to the cluster center ( 5 arcmin) than the F97 field,

We have already purged the star list of stars with poor profile fit and elliptical shape parameters, as determined by the DAOPHOT PSF fitting, and have also cut-off stars with standard errors (as produced by ALLSTAR) greater than twice the median standard error of all stars in the sample, in one magnitude wide bins. For stars on the MS, the cutoff corresponds to a standard error $\sigma(B-V)=(0.06,0.08,0.10,0.15)$ mag. at $V=(20,21,22,23)$ mag. respectively. We call this set of stars "sample one", and it corresponds to what is shown in Figure 5. We then further excluded all stars with standard errors greater than $0.030 \mathrm{mag}$, which had the effect of removing all stars fainter than $V=22.6$ as well as the significant number of stars with color errors between 0.03 mag and the cut-offs given above. The remaining stars are "sample two". The radial distributions of the stars in the two samples differ negligibly, with less than one pixel difference in mean distance from the cluster center. We then compared the color distributions across the same MS fiducial, from $V=20.0-22.0$, normalizing the two distributions at the peak. The 
normalizing factor (1.16) is not too different from unity, and the resulting distributions are plotted in Figure 7. Bluewards of the peak the two curves are nearly identical. The redward distributions are very different, with the low error plot (sample 2) showing substantially fewer stars and a distribution close to symmetrical across the peak, especially if the field star contributions are taken into account. Given the ADDSTAR experiments discussed above, some of small number of excess stars (the red side of the sample two distribution) may be optical binaries, but even if all the outliers are physical binaries they contribute no more than $10 \%$ of the total number of stars measured on the MS. The distribution is however, equally consistent with a MS binary proportion of zero.

These experiments show that within the annulus under consideration, the apparent binary population is due to a non-Gaussian distribution of color errors about the MS fiducial. An explanation could be that the most likely color of any contaminating star is redder than a given MS star, since fainter stars are either cool cluster MS stars or very red foreground dwarfs, or faint compact galaxies. We therefore conclude that there is no clear evidence from the MS color distribution to support the existence of a large proportion of physical binaries in NGC 2808, at least outside a radius of a few arcmin.

\section{FINAL REMARKS}

NGC 2808 has the most unusual HB morphology of any galactic GC, and is an important fiducial for any explanation of the late stages of stellar evolution in general and in high-density GC in particular. In this paper, based on photometrically secure observations, we determine the cluster reddening to be $E(B-V)=0.20 \pm 0.02$ and show that this is consistent with the spectroscopically determined metallicity. Small differences in the values for $E(B-B)$ and $[\mathrm{Fe} / \mathrm{H}]$ via different methods may only reflect calibration difficulties, but may also be indicative of differences in $[\alpha / F e]$ between the comparison clusters chosen to have similar $[\mathrm{Fe} / \mathrm{H}]$ to NGC 2808. Significant progress in this regard requires high dispersion spectroscopy in order to determine total metal abundance $[M / H]$. The CMD presented confirms and extends recent HST results, although there is an 0.05 mag difference in the $B-V$ photometric zeropoint. No significant differences in radial gradients between various groups of stars on the CMD are found, while the presence of a large fraction of MS binaries is not confirmed. However the observations here do not extend into the core region of this very dense cluster, and studies of gradients and binary fraction to the very center of NGC 2808, using HST, would be of considerable interest. 


\section{REFERENCES}

Alcaino, G., \& Liller, W. 1986, AJ, 91, 87

Alcaino, A., Liller, W., Alvarado, F., \& Wenderoth, E. 1990, ApJS, 72, 693 (A90)

Armandroff, T.E., \& Zinn, R. 1988, AJ 96, 92

Borassova, J., Catelan, M., Spassova, N., \& Sweigart, A.V. 1997, AJ, 113, 692

Brocato, E., Castellani, V., Scotti, G.A., Saviane, I., Piotto, G., \& Ferraro, F.R. 1998, A\&A, 335, 929

Brocato, E., Castellani, V., Raimondo, G., \& Walker, A.R. 1999, MNRAS, in press

Buonanno, R., Corsi, C.E., \& Fusi Pecci, F. 1989, A\&A, 216, 80

Burstein, D, \& Heiles, C. 1984, ApJS, 54, 33

Byun, Y-L., \& Lee, Y-W. 1993, in ASP. Conf. Ser. 13, "The Formation and Evolution of Star Clusters", ed. K. Janes (San Francisco: ASP), 243

Carretta, E., \& Gratton, R.G. 1997, A\&AS, 121, 95

Cassisi, S., \& Salaris, M. 1997, MNRAS, 285, 593

Clement, C., \& Hazen, M. 1989, AJ, 97, 414

Da Costa, G.S., \& Armandroff, T.E. 1995, A.J., 1092533

Dorman, B., Rood, R.T., \& O’Connell, R.W. 1993, ApJ, 419, 596

Ferraro, F.R., Clementini, G., Fusi Pecci, F., Buonanno, R., \& Alcaino, G. 1990, A\&AS, 84,59

Ferraro, F.R., Carretta, E., Fusi Pecci, F., \& Zamboni, A. 1997, A\&A, 327, 598 (F97)

Ferraro, F.R., Paltrinieri, B., Fusi Pecci, F., Rood, R.T., \& Dorman, B. 1998, ApJ, 500, 311

Gratton, R.G., \& Ortolani, S. 1986, A\&AS, 65, 63

Harris, W.E. 1974, ApJ, 192, L14

Harris, W.E. 1975, ApJS, 29, 397

Harris, W.E. 1978, PASP, 90, 45

Holtzman, J.A., et al. 1995, PASP, 107, 1065

Iben, I., Jr. 1968, Nature, 220, 143

Landolt, A.U. 1992, AJ, 104, 340

Mighell, K.J., Sarajedini, A., \& French, R.S. 1998, AJ, 116, 2395

Rubenstein, E.P., \& Bailyn, C.D. 1999, ApJ, 513, L33 
Rutledge, G.A., Hesser, J.E., Stetson, P.B., Mateo, M., Simard, L., Bolte, M., Friel, E.D., \& Copin, Y. 1997a, PASP, 109, 883

Rutledge, G.A, Hesser, J.E., \& Stetson, P.B. 1997b, PASP, 109, 907

Salaris, M., Chieffi, A., \& Straniero, O. 1993, ApJ, 414, 580

Sarajedini, A., \& Layden, A. 1997, AJ, 113, 264

Saviane, I., Piotto, G., Fagotto, F., Zaggia, S., Capaccioli, M., \& Aparicio, A. 1998, ApJ, 414,580

Schlegel, D.J., Finkbeiner, D.P., \& Davis, M. 1998, ApJ, 500, 525

Sosin, C., et al. 1997, ApJ, 480, L35 (S97)

Stetson, P.B. 1987, PASP, 99, 191

Sturch, C.R. 1966, ApJ, 143, 774

Stetson, P.B. 1995, "DAOPHOT II User's Manual" (Victoria: Dominion Astrophys. Obs.)

Vandenberg, D.A., \& Bell, R.J. 1985, ApJS, 58, 561

Walker, A.R. 1990, AJ, 100, 1532

Walker, A.R. 1992, PASP, 104, 1063

Walker, A.R. 1995, PASP, 107, 683

Walker, A.R. 1998, AJ, 116, 220

Walker, A.R. 1999, in preparation

Zinn, R.J. 1985, ApJ, 293, 424

Zinn, R.J., \& West, M.J. 1984, ApJS, 55,45 
Fig. 1.- NGC 2808, with the local standards identified, from a 5 second V band exposure with the CTIO 4-m PFCCD camera. The field size is 14.75 x 14.75 arcmin, with north to the right and east up. The scale units are 4 pixels, or 1.728 arcsec.

Fig. 2.- Color-magnitude diagram for all stars in the 4-m field. The data plotted are a combination of measurements on the short, medium and long exposures, using a weighted mean for cases of multiple measurement. The photometry has been corrected for differential reddening with respect to the center of the field.

Fig. 3.- Color-magnitude diagram for stars closer than 350 pixels (2.5 arcmin) from the center of NGC 2808.

Fig. 4.- Color-magnitude diagram for stars more than 1000 pixels (7.2 arcmin) distant from the center of NGC 2808.

Fig. 5.- Color-magnitude diagram for stars measured within an annulus of radii 600 and 800 pixels (4.3 and 5.8 arcmin) centered on NGC 2808.

Fig. 6. - Color-magnitude diagram for stars measured within an annulus of radii 800 and 1000 pixels (5.8 and 7.2 arcmin) centered on NGC 2808.

Fig. 7.- Normalized histograms showing the distribution of stars around a fiducial centered on the MS, from $V=20-22$. See text for details. 


\section{TABLE CAPTIONS}

Table 1: NGC 2808 Local Standards

Table 2: HB Color Location 
Table 1. NGC 2808 Local Standards

\begin{tabular}{lllll}
\hline \hline Num & \multicolumn{1}{c}{$\mathrm{X}$} & \multicolumn{1}{c}{$\mathrm{Y}$} & \multicolumn{1}{c}{$V$} & $B-V$ \\
\hline 1 & 1043.94 & 548.13 & 13.544 & 0.942 \\
2 & 1234.07 & 276.91 & 14.425 & 1.136 \\
3 & 1020.65 & 507.75 & 15.091 & 1.134 \\
4 & 1416.60 & 197.26 & 15.848 & 0.915 \\
5 & 189.81 & 1749.83 & 13.444 & 1.930 \\
6 & 167.40 & 1879.44 & 14.747 & 0.436 \\
7 & 451.61 & 1712.06 & 13.933 & 1.077 \\
9 & 282.54 & 1545.45 & 13.085 & 0.569 \\
10 & 58.69 & 967.08 & 14.919 & 0.586 \\
11 & 218.19 & 514.51 & 15.737 & 0.882 \\
12 & 457.55 & 264.05 & 14.591 & 0.696 \\
13 & 1007.26 & 122.55 & 14.417 & 1.384 \\
14 & 771.75 & 41.31 & 14.318 & 1.409 \\
15 & 1581.32 & 76.12 & 13.627 & 1.271 \\
16 & 1839.59 & 219.65 & 13.389 & 0.476 \\
18 & 1783.94 & 454.64 & 14.613 & 1.287 \\
19 & 1995.65 & 871.26 & 13.951 & 0.609 \\
21 & 1855.74 & 1684.73 & 14.423 & 0.852 \\
23 & 1063.22 & 1779.47 & 13.841 & 0.833 \\
\hline
\end{tabular}

Table 2. HB Color Location

\begin{tabular}{llll}
\hline \hline \multicolumn{1}{c}{ Source } & RHB & BHB & Difference \\
\hline This paper & 0.83 & 0.13 & 0.70 \\
Sosin et al & 0.78 & 0.08 & 0.70 \\
Ferraro & 0.88 & 0.17 & 0.71 \\
Alcaino & 0.80 & - & - \\
Byun \& Lee & 0.82 & 0.06 & 0.76 \\
\hline
\end{tabular}


This figure "figure1.jpg" is available in "jpg" format from: http://arxiv.org/ps/astro-ph/9904387v1 
This figure "figure2.jpg" is available in "jpg" format from: http://arxiv.org/ps/astro-ph/9904387v1 
This figure "figure3.jpg" is available in "jpg" format from: http://arxiv.org/ps/astro-ph/9904387v1 
This figure "figure4.jpg" is available in "jpg" format from: http://arxiv.org/ps/astro-ph/9904387v1 
This figure "figure5.jpg" is available in "jpg" format from: http://arxiv.org/ps/astro-ph/9904387v1 
This figure "figure6.jpg" is available in "jpg" format from: http://arxiv.org/ps/astro-ph/9904387v1 
This figure "figure7.jpg" is available in "jpg" format from: http://arxiv.org/ps/astro-ph/9904387v1 